\title{
Extension de la plage de fonctionnement à rendement élevé des alimentations à découpage ${ }^{1}$
}

\author{
François Costa ${ }^{1,2}$, Alain Cunière ${ }^{1,3}$. \\ ${ }^{1}$ SATIE (UMR 8029), ENS de Cachan PRES UniverSud, 61, Av. du Président Wilson, 94230 Cachan \\ ${ }^{2}$ IUFM de Créteil, université Paris 12, place du 8 mai 1945, 93000 Saint Denis \\ ${ }^{3}$ Lycée Pierre de Coubertin, Chaussée de Paris, 77100 Meaux,
}

Résumé-L'introduction des techniques de conversion statique a permis un gain spectaculaire du rendement des alimentations continues. Toutefois, de nouvelles contraintes apparaissent qui contribuent à dégrader les résultats obtenus, nécessitant ainsi de nouveaux efforts pour poursuivre l'amélioration. En effet, on constate que la généralisation des techniques numériques et l'intégration croissante a mené à un abaissement des tensions à fournir avec un accroissement correspondant des courants, facteurs défavorables au rendement. La nécessité de miniaturisation a conduit à élever les fréquences de découpage, contribuant aussi à la dégradation du rendement. Enfin, la dissémination de ces technologies dans les domaines de consommation de masse impose la nécessité de réduire la consommation de veille ou à très faible charge. Cet article se propose de faire, dans un premier temps, un point sur l'origine des pertes d'un convertisseur, puis de proposer quelques pistes d'innovation pour la diminution des pertes.

Mots clés - réduction des pertes, convertisseurs très basse tension, structures multicellulaires, inductance point milieu, structures distribuées, matériaux magnétiques, packaging.

\section{INTRODUCTION}

L'accroissement des performances de rendement d'un convertisseur statique avec ses régimes de marche reste un enjeu d'importance même si des progrès considérables ont été réalisés comme on le verra plus loin. En effet, une meilleure compréhension et une gestion améliorée des pertes permet d'intervenir à plusieurs niveaux :

- avoir une approche globale des pertes d'un convertisseur au stade de la conception qui intègre son mode de fonctionnement et son usage dans la durée et non plus en un seul point particulier (le nominal). Cette réflexion a des conséquences sur le choix de la structure de conversion, des composants, des dispositifs et des modes de commande.

- améliorer le rendement des dispositifs d'alimentation très basse tension et très fort courant (de l'ordre de 70 à $80 \%$ ) qui sont actuellement en diffusion rapide (processeurs, éclairage à LED, pile à combustible, véhicule hybride, etc..). Il est important de conserver un rendement élevé sur toute la plage de fonctionnement en charge du convertisseur, le problème se posant surtout à faible charge où le rendement devient généralement mauvais,

${ }^{1}$ Cet article, avant relecture et correction pour la revue J3EA, a fait l'objet d'une communication aux journées 2007 de la section électrotechnique sur le thème Energie et Développement Durable à l'antenne de Bretagne de l'ENS de Cachan les 14 et 15 mars 2007.
- permettre la miniaturisation des dispositifs : si on réduit les pertes ou qu'on recherche leur optimisation globale, les dispositifs de drainage thermique le seront aussi et in fine le volume total; l'intégration en électronique de puissance résulte de cette idée.

\section{BILAN DES PERTES DANS UNE CELLULE DE COMMUTATION}

Pour illustrer l'origine des pertes dans un convertisseur, nous allons considérer une cellule de commutation, comme représenté à la figure 1. Elle comprend des composants actifs, passifs et parasites qui tous vont contribuer aux pertes de la cellule.

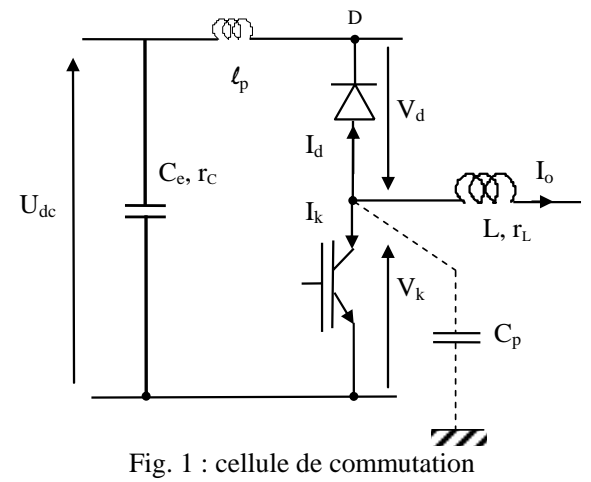

On définit les grandeurs suivantes :

$U_{d c}$ : tension d'alimentation de la cellule, $\mathrm{I}_{0}$ : courant de sortie

$r_{d s o n}$ : résistance à l'état passant de l'interrupteur commandé

$r_{d}$ : résistance dynamique de diode

$V_{j}$ : tension d'interrupteur à l'état passant

$V_{f}$ : tension directe de diode

$E_{d}$ : énergie dissipée au blocage de la diode, $\mathrm{Q}_{\mathrm{rr}}$ : charge recouvrée

$I_{r r m}$ : courant de recouvrement inverse de diode,

$t_{o n}, t_{\text {off }}$ : temps de commutation à la mise en conduction et au blocage

$E_{\text {ton }}$ : énergie dissipée à la fermeture de l'interrupteur

$E_{\text {toff }}$ : énergie dissipée au blocage de l'interrupteur

$V_{k}, I_{k}$, respectivement tension et courant d'interrupteur,

$Q_{g s}$ : charge de grille, $V_{g s}$ : tension de grille, $C_{g s}$ : capacité de grille

$F_{S w}:$ fréquence de commutation

$B_{M}$ : induction maximale dans l'inductance, $r_{L}\left(F_{s w}\right)$ résistance de pertes Joule du bobinage, elle dépend de la fréquence.

$r_{C}$ : résistance de pertes Joule du condensateur

$\ell_{\mathrm{p}}$ : inductance parasite de câblage de la maille de commutation, $C_{p}$ : capacité parasite globale à la terre

L'expression des pertes peut être donnée sous la forme suivante : 
$P_{\text {total }}=P_{\text {cond }}+P_{\text {sw }}+P_{g}+P_{\text {passif }}+P_{\text {paras }}$

Où :

$-\mathrm{P}_{\text {cond }}$ exprime les pertes par conduction :

$P_{\text {cond }}=k_{1} r_{\text {dson }} I_{o}^{2}+k_{2} r_{d} I_{o}^{2}+k_{3} V_{j} I_{o}+k_{4} V_{f} I_{O}$

Les coefficients $k_{i}$ dépendent de la forme du courant dans l'interrupteur,

$-\mathrm{P}_{\mathrm{sw}}$ exprime les pertes par commutation dans les interrupteurs :

$P_{\text {sw }}=F_{\text {sw }}\left(E_{d}+E_{\text {ton }}+E_{\text {toff }}\right)$

Avec :

$E_{\text {ton }}+E_{\text {toff }} \propto\left(t_{\text {on }}+t_{\text {off }}\right) I_{k} V_{k}$

et :

$E d \propto\left(Q_{r r}\right)_{I_{k}} U_{d c}$

$-\mathrm{P}_{\mathrm{g}}$ exprime les pertes dans la grille de l'interrupteur, celle-ci se charge et se décharge à chaque commutation, au final :

$P_{g}=F_{s w} Q_{g s} V_{g s}$

$-\mathrm{P}_{\text {passif }}$ exprime les pertes dans les composants passifs, on ne considérera ici que les pertes hystérésis et par effet Joule dans l'inductance et dans le condensateur :

$$
P_{\text {passif }}=a_{1} F_{s w}^{\alpha} B_{M}^{\gamma}+a_{3} r_{L}\left(F_{s w}\right) I_{o}^{2}+a_{4} r_{C} I_{o}^{2}
$$

$1<\alpha, \gamma<2$ selon les types de matériaux ferrite

$-\mathrm{P}_{\text {paras }}$ exprime les pertes dues aux composants parasites de la structure :

$P_{\text {paras }} \propto F_{\text {sw }}\left(\ell_{p} I_{o}^{2}+C_{p} U_{d c}{ }^{2}\right)$

Ainsi, on constate donc qu'il est possible de classer les facteurs déterminant les pertes selon plusieurs catégories :

-ceux liés à la charge et au régime de commutation : $I_{o}, V_{k}$, $I_{k}$, en commutation douce, les grandeurs $V_{k}$ et/ou $I_{k}$ sont nulles (ou très faibles),

-ceux liés aux contraintes de conception et à la structure du convertisseur : $U_{d c}, F_{s w}, V_{g s}$,

-ceux découlant des choix technologiques: $B_{M}, r_{L}, r_{C}, r_{d s o n}$, $r_{d}, Q_{r r}, Q_{g}, \ell_{p}, C_{p}$,

On peut toutefois noter que le courant de charge à fournir et la fréquence de découpage constituent des termes très sensibles d'accroissement des pertes pour plusieurs termes : pertes magnétiques, par effet Joule, par commutation et dans la commande.

On va donc présenter dans la suite différentes techniques permettant d'accroître le rendement de conversion en référence aux points qui ont été évoqués ci-dessus.

\section{INFLUENCE DU COURANT DE CHARGE ET DU MODE DE COMMUTATION}

\section{A. Mode de commutation et répartition des pertes dans les semi-conducteurs}

En commutation commandée, le courant de charge $I_{o}$ issu de la cellule de commutation détermine fortement les pertes par conduction par commutation et dans l'inductance; nous avons $I_{k}=I_{o}+I_{r r m}$ et $V_{k}=U_{d c}$, les pertes par commutation sont significatives. Toutefois, si la cellule opère en commutation douce, ces pertes peuvent être significativement diminuées car $I_{k}$ (ou $V_{k}$ ) est généralement très faible. Cependant la mise en œuvre de la commutation douce impose l'utilisation de composants réactifs et éventuellement d'interrupteurs auxiliaires qui génèrent aussi des pertes : le gain au bilan global n'est pas toujours significatif. Ainsi, le choix de la structure de conversion et de son mode de commutation selon son usage sont-ils primordiaux. A titre d'illustration on a comparé [1] au tableau 1 la répartition des pertes au point nominal dans un absorbeur sinusoïdal BOOST (voir les spécifications sur la figure 2) que l'on peut aussi faire fonctionner en mode ZVS [2] moyennant l'adjonction d'un auxiliaire (en gris sur la figure 2) et toutes choses égales par ailleurs (câblage, composants etc..).

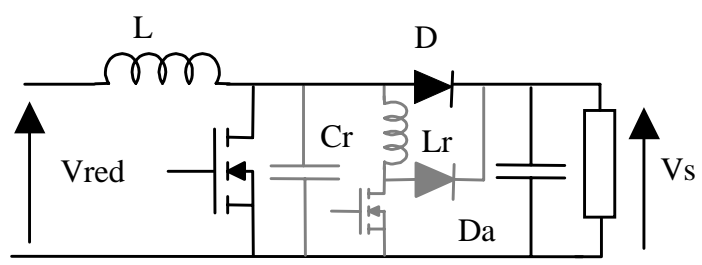

$\mathrm{T}$

Ta

Fig. 2. Structure BOOST en commutation commandée, et en mode ZVS avec les auxiliaires en gris

Alimentation réseau $240 \mathrm{~V}$, tension de sortie $\mathrm{Vs}=400 \mathrm{~V}, \mathrm{P}_{\mathrm{s} \text { nominale }}=1500 \mathrm{~W}$

\begin{tabular}{lll}
\hline Ps=1500W & BOOST commandé & BOOST ZVS \\
\hline MOSFET & $19 \mathrm{~W}$ & $7,9 \mathrm{~W}$ \\
Diode & $10 \mathrm{~W}$ & $4,9 \mathrm{~W}$ \\
Redresseur d'entrée & $5,3 \mathrm{~W}$ & $5,7 \mathrm{~W}$ \\
Autres+auxiliaires & $22,7 \mathrm{~W}$ & $23,5 \mathrm{~W}$ \\
\hline Total & $57 \mathrm{~W}$ & $42 \mathrm{~W}$ \\
\hline
\end{tabular}

TABLEAU 1 : COMPARAISON DES PERTES SELON LE MODE DE COMMUTATION

Le tableau montre bien l'intérêt global de la commutation en mode ZCS pour la cellule principale de commutation. Toutefois si on change de point de fonctionnement, on constate qu'à faible charge (Ps $<450 \mathrm{~W}$ ) le bilan est moins favorable pour le fonctionnement en mode ZVS comme le montre les courbes de rendement de cette structure.

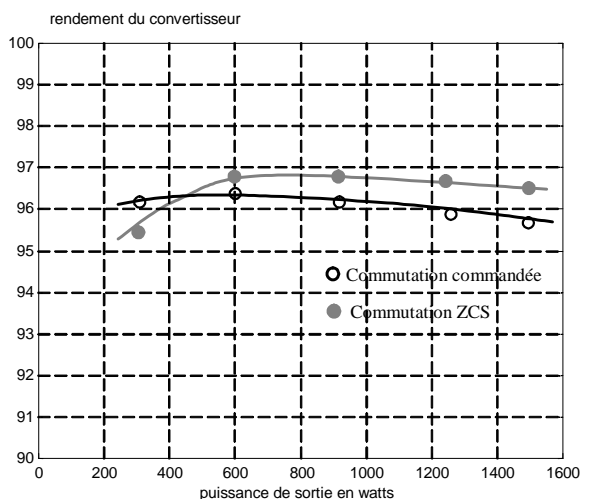

Fig. 3. Evolution du rendement avec la puissance de sortie

Ainsi, une solution possible pour concilier l'intérêt des deux modes est-il de concevoir un mode de commande mixte selon la puissance. Il en résulte un surcoût économique (les auxiliaires) qui peut être évalué au regard du gain énergétique réalisé à faible charge et à l'économie en coût d'exploitation qu'il représente selon le contexte d'usage du dispositif.

\section{B. Adaptation du calibre de l'interrupteur au courant de charge}

De façon plus générale, il est intéressant de chercher à moduler les pertes selon le niveau de charge. Ainsi, si on 
constitue un interrupteur par n transistors MOSFET calibrés à $I_{0} / n$ en parallèle, commutant à haute fréquence, les pertes totales s'expriment par :

$$
P_{\text {MOSFET }}=n \cdot\left[k_{1} r_{d s o n}\left(\frac{I_{o}}{n}\right)^{2}+k_{2} F_{s w} \frac{I_{o}}{n}+Q_{g} V_{g s} F_{s w}\right]
$$

Cette fonction admet un minimum pour :

$$
n_{\text {opt }}=\sqrt{\frac{k_{1} r_{d s o n}}{Q_{g} V_{g s} F_{s w}}} I_{o}
$$

On constate donc l'intérêt d'adapter (au moins par saut) le nombre d'interrupteurs proportionnellement au courant de sortie à cause des pertes dans la grille à haute fréquence. Cette solution est évidemment lourde avec des composants discrets mais devient simple de mise en œuvre avec des composants de puissance cellulaires qui intègrent leur commande rapprochée [3], l'évolution technologique en termes d'intégration de puissance va dans ce sens.

\section{Structure de conversion : cas des alimentations TBT}

Une problématique spécifique concerne actuellement les alimentations DC-DC très basse tension destinées aux systèmes électroniques numériques (informatique, télécommunications), à la domotique ou à l'éclairage à LED. Usuellement, le rendement de ces dispositifs ne dépasse guère 80 (consommation de la commande comprise), il reste donc des marges significatives de gain de rendement.

Plusieurs facteurs influent dans cette problématique :

- l'abaissement des tensions d'alimentation des composants numériques [4] due à l'accroissement de la densité d'intégration et à la montée en fréquence d'horloge (processeurs à $3 \mathrm{GHz}$ ), le corollaire est l'accroissement des courants consommés, actuellement un processeur alimenté sous $1,2 \mathrm{~V}$ peut consommer jusqu’à $70 \mathrm{~A}$ ! Une évolution assez similaire apparaît avec l'éclairage à LED blanches : il est nécessaire de les alimenter à basse tension et relativement fort courant (par LED : 3,3V 350mA @80 lumen) pour constituer des sources lumineuses puissantes. Les solutions actuelles sont basées sur l'utilisation de hacheurs abaisseurs entrelacés et de redresseurs synchrones (et leur dispositif de commande adapté) [5] , comme représenté à la figure 4, la chute de tension dans des diodes Schottky serait est en effet trop pénalisante.

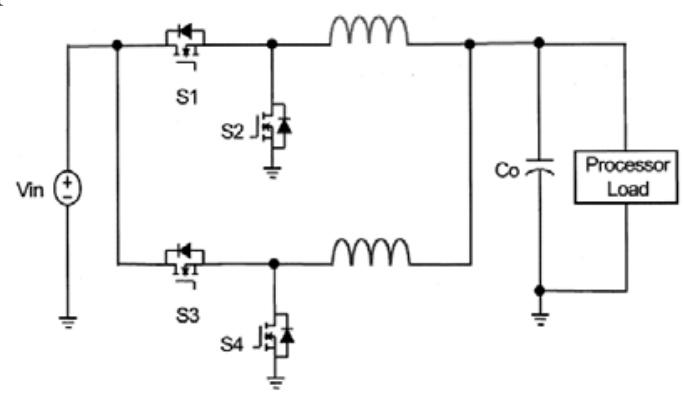

Fig. 4. Hacheur abaisseur entrelacé à 2 cellules

- la recherche de miniaturisation : elle va de pair avec les fonctions avancées de l'électronique numérique et elle est impérative dans les dispositifs nomades ou dans les systèmes à forte compacité (aéronautique, automobile). Cette évolution, croisée avec la précédente lorsque l’on recherche des dynamiques de contrôle très élevées, impose donc d'accroître les fréquences de commutation pour diminuer la taille des composants passifs ; elles se situent actuellement au-delà de $100 \mathrm{kHz}$ et des produits fonctionnant autour de $1 \mathrm{MHz}$ commencent à apparaître.

Ainsi se pose la question du rendement de structures de conversion à fort facteur d'abaissement de la tension en sortie.

Usuellement, la très basse tension de sortie est fournie par un hacheur abaisseur et se pose alors la question du choix de sa tension d'entrée. En effet, à tension et courant de sortie $V_{o}, I_{o}$ donnés, le hacheur aura des pertes en conduction et commutation d'autant plus faibles que le rapport cyclique sera grand et la tension d'entrée $U_{d c}$ faible :

$$
\begin{aligned}
& \text { - Sous } U_{d c} \text { : } \\
& P_{\text {transistor }}=r_{d s o n} I_{o}^{2} \sqrt{\alpha}+I_{o} U_{d c}\left(t_{o n}+t_{o f f}\right) F_{s w} / 2
\end{aligned}
$$

Si on divise par un facteur $\mathrm{k}$ cette tension, les pertes deviennent (à supposer que les temps de commutation ne varient pas) :

- Sous $U_{d c} / k$ :

$$
P_{\text {transistor }}=r_{d s o n} I_{o}^{2} \sqrt{k \alpha}+\frac{I_{o} U_{d c}}{k}\left(t_{o n}+t_{\text {off }}\right) F_{s w} / 2
$$

Les pertes par conduction sont accrues d'un facteur $\sqrt{k}$ mais les pertes par commutation (dominantes en HF) sont divisées par $k$. Au bilan et pour les convertisseurs évoqués qui fonctionnent nécessairement à haute fréquence, le gain est substantiel. Une étude de rendement effectuée dans le cas d'une structure de hacheur abaisseur entrelacés montre que le gain de rendement est très significatif avec l'abaissement de la tension d'alimentation.
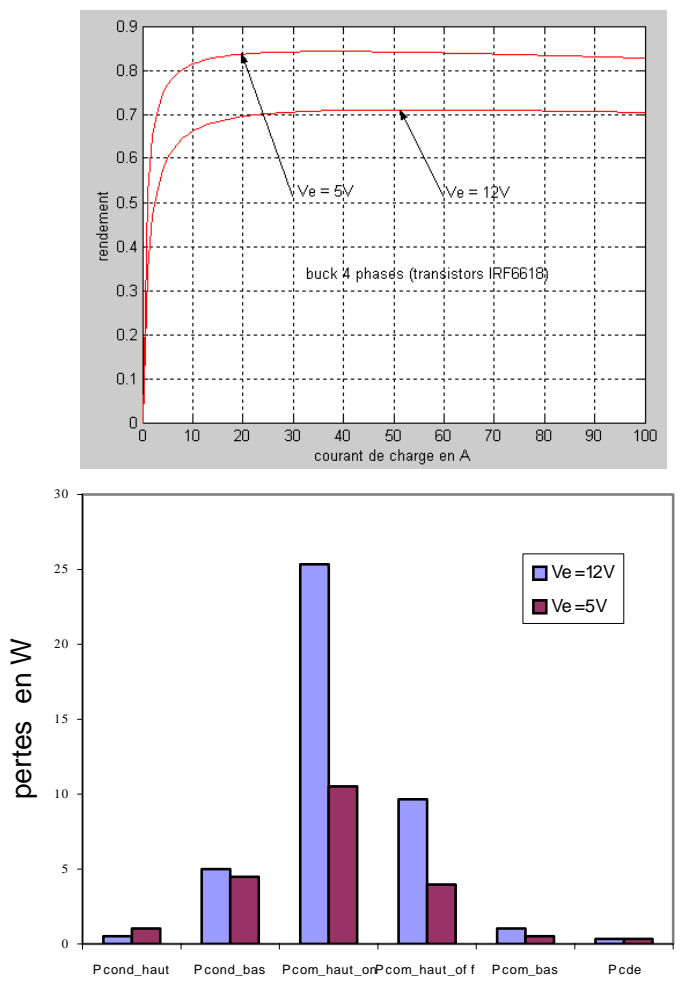

Fig. 5. Rendement et pertes dans les composants selon la tension d'alimentation 
L'analyse des pertes dans cette structure montre qu'elles sont importantes en commutation pour l'interrupteur haut et en conduction pour l'interrupteur bas ; ceci étant dû à la très faible valeur du rapport cyclique qui impose des contraintes spécifiques aux deux interrupteurs.

\section{1) Structure de conversion non isolée permettant l'amélioration du rendement [6]}

\section{a) Structure permettant de réduire les contraintes sur} les interrupteurs

Dans l'idée d'amélioration du rendement de la structure Buck fonctionnant à faible rapport cyclique, il est possible de réduire la contrainte en courant dans l'interrupteur K2 en utilisant une prise intermédiaire sur l'inductance de sortie comme représenté à la figure 6.

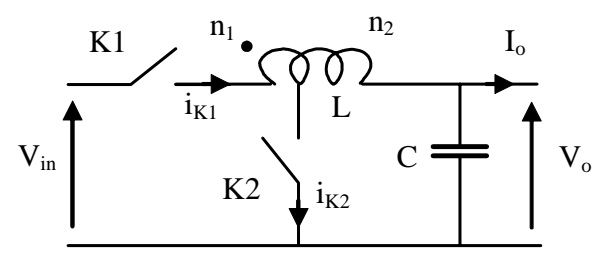

Fig. 6. Hacheur de Buck à prise intermédiaire sur l'inductance de lissage

En considérant que les $\mathrm{n}_{2}$ spires forment l'inductance $L$ de sortie (conduction de K2), il apparaît que pendant la phase de stockage d'énergie (conduction de K1), l'inductance devient $n^{2} L$ avec :

$n=\frac{n_{1}+n_{2}}{n_{2}}$

A même énergie stockée, le courant nécessaire sera divisé par $n$ et le temps nécessaire pour stocker cette énergie multiplié par $n$. L'accroissement du rapport cyclique est donné par la relation :

$\frac{V_{0}}{V_{\text {in }}}=\frac{\alpha}{\alpha+n \cdot(1-\alpha)}$

La figure 7 montre le résultat obtenu pour différentes valeurs du rapport $n$ par comparaison avec le classique hacheur Buck (courbe rouge). Le principe d'entrelacement peut évidemment s’appliquer à cette structure.

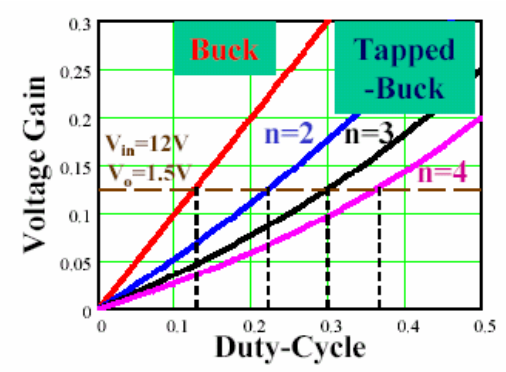

Fig. 7. Allongement du rapport cyclique avec la valeur de $n$ pour un gain en tension donné (d’après [6])

Les contraintes en tension et courant sur les différents interrupteurs deviennent :

$V_{K 2}=\frac{V_{\text {in }}-V_{o}}{n}+V o=\left(\frac{1-G}{n}+G\right) V_{\text {in }}$

avec :

$G=V_{o} / V_{\text {in }}$
$I_{K 2}=I_{o}$

$I_{K 1}=\left(\frac{1-G}{n}+G\right) I_{o}$

$V_{K 1}=V_{\text {in }}+(n-1) V_{o}$

Ce montage permet la réduction des pertes grâce au nouveau profil du courant $i_{K 1}$ qui change les contraintes sur les interrupteurs ; la tension bloquée par K2 est plus faible d'où la réduction des pertes de recouvrement de la diode de K2 et l'utilisation possible d'un transistor très basse tension à faible $r_{d s o n}$. Cependant la contrainte en tension sur K1 est accrue mais son courant diminué. Ainsi la structure augmente certaines contraintes mais sur des grandeurs qui ne contribuent pas fortement à l'accroissement des pertes.

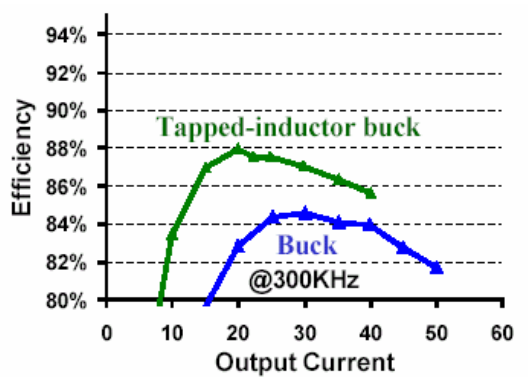

Fig. 8. Amélioration du rendement de par la structure 12-1,5V (d'après [6])

Le rendement est amélioré comme le montre le tracé figure 8 pour un convertisseur 4 phases, $V_{\text {in }}=12 \mathrm{~V} ; \mathrm{V}_{\mathrm{o}}=1,5 \mathrm{~V} ; I_{o}=$ $50 \mathrm{~A}$.

Néanmoins, ce montage présente une surtension de commutation sur K1 due aux imperfections du couplage magnétique. Cet inconvénient est corrigé par l'ajout d'un écrêteur non dissipatif constitué des deux diodes et du condensateur $C_{e}$, comme représenté à la figure 9.

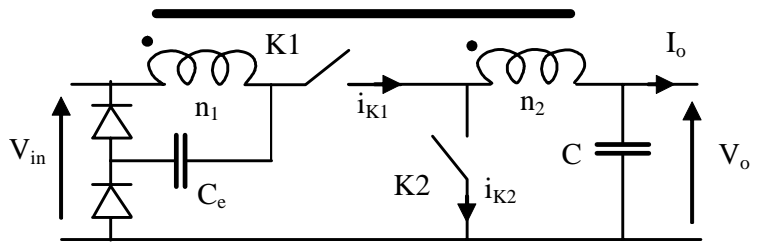

Fig. 9. Structure améliorée (d'après [6])

b) Structure à entrelacement par coupleur magnétique

Le problème inhérent du hacheur de Buck entrelacé à $q$ inductances séparées (fig. 4) réside dans l'importance de l'ondulation de courant dans les inductances. Avec $\frac{\Delta i_{i}}{\left\langle i_{i}\right\rangle}=q^{2} * \frac{\Delta i_{s}}{\left\langle i_{s}\right\rangle}$, il est aisé de conclure qu'à taux d'ondulation donné en sortie du convertisseur, cette technique n'est pas favorable aux pertes dans les inductances et aux pertes par commutation au blocage des transistors.

Des travaux récents [7] ont introduit l’idée de coupler entre elles les inductances de chaque cellule, comme représenté à la figure 10 . Le système $q$-phasé des tensions issues des cellules de commutation $\left(\mathrm{V}_{1}, \mathrm{~V}_{2} \ldots\right)$ est décomposable en composantes de mode différentiel de rang $h \neq k q$ (avec $k=0$, $1,2, \ldots)$ et en composantes de mode commun de rang $h=k q$. 


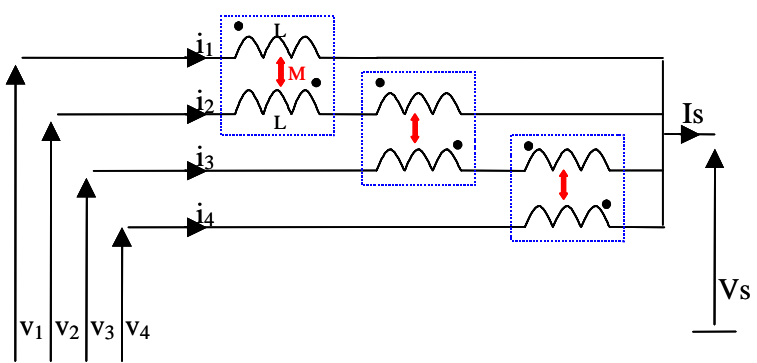

Fig. 10 : structure de hacheur entrelacé avec inductances couplées

Pour la fréquence de découpage $(h=1)$,

$$
\left.\underline{\left(I_{1}\right.}\right)_{1}=\frac{\left(V_{1}\right)_{1}}{2 j L \omega-j M \omega e^{-j \frac{2 \pi}{q}}-j M \omega e^{j \frac{2 \pi}{q}}}
$$

L'inductance magnétisante $L_{\mu}$ correspondante d'expression :

$$
L_{\mu}=2\left[L-M * \cos \left(\frac{2 \pi}{q}\right)\right]
$$

Les courants magnétisants déterminés par $L_{\mu}$ sont négligeables devant les courants de mode commun régis par l'équation :

$$
\frac{\sum_{1}^{q} V_{i}}{q}=2(L-M) \frac{d i_{i}}{d t}
$$

Les courants correspondants sont en phase, ce qui conduit à la relation :

$\frac{\Delta i_{i}}{\left\langle i_{i}\right\rangle}=\frac{\Delta i_{s}}{\left\langle i_{s}\right\rangle}$

Il est à remarquer également que l'entrelacement magnétique multiplie par $q$ la fréquence des courants dans les coupleurs. L'intérêt est clair : à mêmes inductances $L_{f}=2(L-M)$, l'ondulation relative dans les coupleurs est $q^{2}$ plus faible et constitue un avantage très net sur les pertes, ou encore, à même ondulation de courant, les inductances $q^{2}$ plus faibles permettent des dynamiques du courant de sortie plus adaptées aux besoins des alimentations TBT.

L'inductance de stockage correspondant aux deux enroulements d'un coupleur dans la même fenêtre, présente l'avantage d'une distribution unidimensionnelle du champ (figure 11) et ainsi un accroissement moindre de la résistance avec la fréquence, et, par corollaire l'absence d'entrefer. Une permutation particulière des tensions de phase minimise le flux dans les parties magnétiques et indirectement le volume du composant.

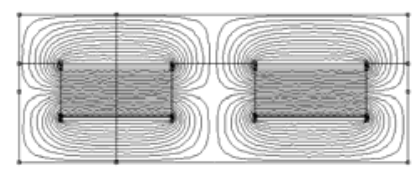

Fig. 11. Répartition du flux de mode commun dans un coupleur

\section{2) Structure de conversion isolée}

Une autre possibilité pour réaliser une forte atténuation de tension entre l'entrée et la sortie d'un convertisseur DC-DC consiste à utiliser un transformateur abaisseur, avec toutefois l'introduction d'une nouvelle source de pertes. Un exemple de structure à doubleur de courant en commutation commandée [8] bien adaptée à la basse tension et aux forts courants de sortie est proposé à la figure 12. Le schéma est présenté accompagné des formes d’onde caractéristiques.

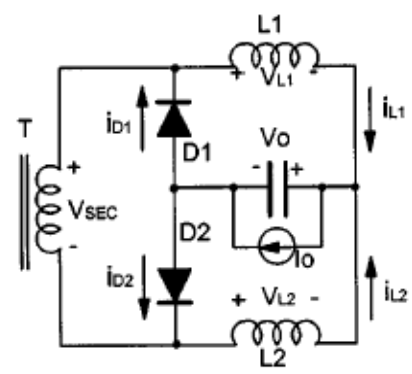

a) fréquence $i_{L}: F_{s w}$, courant moyen $\mathrm{I}_{\mathrm{o}} / 2$ Fréquence $\mathrm{i}_{\mathrm{C}}: 2 F_{\mathrm{sw}}$

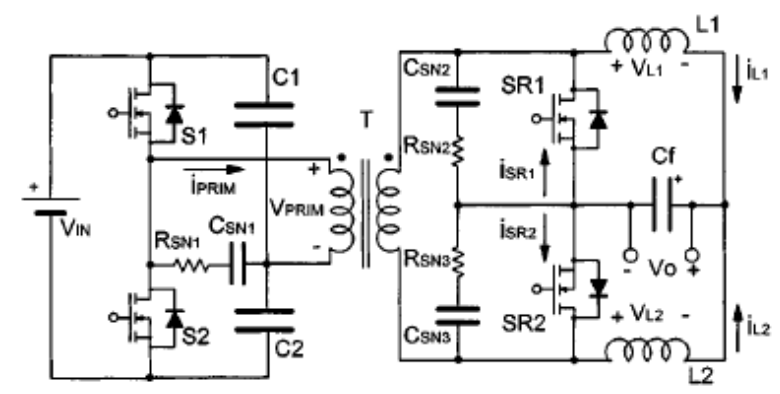

b)

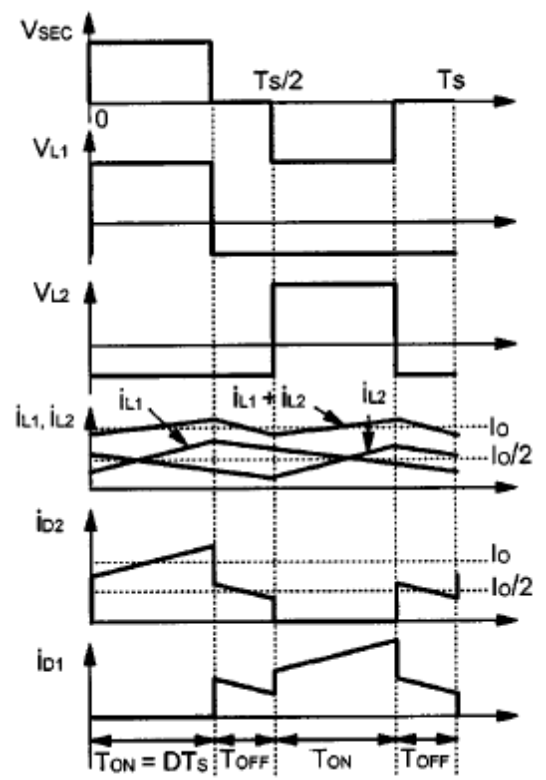

Fig. 12. Structure à redresseur à doublement de courant

Les courants d'inductance sont entrelacés: chaque inductance est parcourue par le courant moyen $I_{\delta} / 2$ et soumise à $V_{s e c}-V_{0}$ et $-V_{0}$. à la fréquence $F_{s w}$, ce qui est plus favorable en termes de pertes magnétiques et Joules dans les enroulements. Le condensateur voit donc une ondulation de courant divisée par 2 en amplitude et à la fréquence $2 F_{s w}$, d'où un gain d'un facteur 2 par rapport au cas précédent.

Par ailleurs, il est possible d'intégrer l'ensemble des composants inductifs sur un seul circuit magnétique pour réduire l'encombrement la connectique et les pertes [9].

Par ailleurs, la structure primaire en demi-pont à point milieu capacitif est bien adaptée pour réduire les pertes dans les semi-conducteurs primaires : ceux-ci n'ont à supporter que la demi-tension d'alimentation, ce qui est favorable vis-à-vis des pertes en commutation. Enfin la présence du pont capacitif permet de s'affranchir d'éventuelles dissymétries de commande qui tendraient à saturer le transformateur. 
3) La topologie des systèmes d'alimentation: de l'alimentation continue au système d'alimentation DC

Le concept même d'alimentation DC-DC est en train d'évoluer : nous passons d'un dispositif central et souvent multi-sorties à un système de convertisseurs distribués constitué par de multiples étages, isolés ou non, chacun ayant des performances spécifiques (ondulation, réponse dynamique, rendement) adaptées à sa localisation et aux performances à fournir. La figure 13 présente cette évolution.
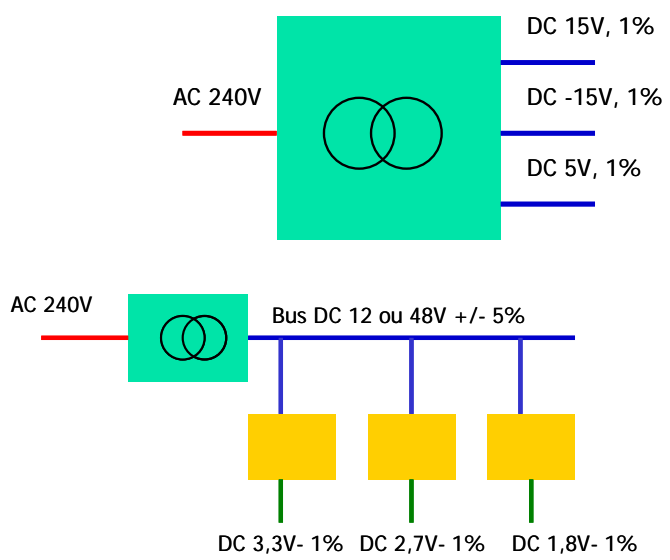

Figure 13 : évolution de la topologie d’alimentation

On a détaillé ci-dessous pour chaque solution les avantages et inconvénients : il apparaît que la solution d'alimentation distribuée présente plus d'avantages que la solution centralisée au détriment du coût, mais celui-ci peut varier avec la diffusion de masse !

\section{SOLUTION CENTRALISEE}

Avantages

Simplicité, coût, solutions éprouvées

Isolation galvanique, régulation aisée

Inconvénients

Défaillance généralisée en cas de défaut sur une des sorties

Structure figée

système alimenté

Coût élevé si nécessité de redondance

Gestion de la thermique centralisée

critique parfois

Problèmes en cas de connectique de sortie

longue

Rendement faible pour les très faibles

tension de sortie $(<80 \%)$
SOLUTION DISTRIBUEE

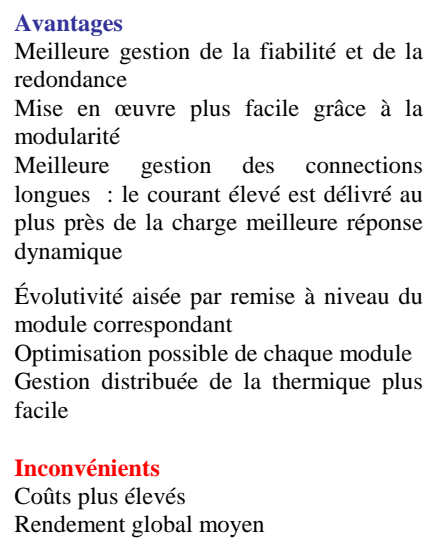

Meilleure gestion de la fiabilité et de la redondance

Mise en œuvre plus facile grâce à la modularité

Meilleure gestion des connections

longues : le courant élevé est délivré au plus près de la charge meilleure réponse dynamique

Évolutivité aisée par remise à niveau du module correspondant

Optimisation possible de chaque module Gestion distribuée de la thermique plus facile

Inconvénients

Coûts plus élevés

Rendement global moyen

TABLEAU 2 : COMPARAISON DES PERFORMANCES

La structure des alimentations distribuées est souvent organisée en trois niveaux :

- un étage d'entrée (front end) qui distribue un bus DC moyenne tension $(48 \mathrm{~V})$ à absorption sinusoïdale, la régulation de tension est médiocre à ce niveau.

- un étage intermédiaire DC-DC qui abaisse la tension à 12 ou $5 \mathrm{~V}$, la qualité de tension est meilleur ainsi que la dynamique,

- un étage final qui alimente directement l'application spécifique sous très basse tension (de 1 à 3,3V) avec des performances dynamiques et d'ondulation maximales (PoL converter : convertisseur au point d'alimentation) [10].

L’isolement galvanique peut être localisé aux différents niveaux évoqués comme représenté à la figure 14. La topologie b) à l'intérêt de créer une isolation galvanique entre les différentes applications finales, point qui peut avoir son intérêt pour limiter la circulation de courants parasites entre elles. Son coût est évidemment plus important.

L'autre intérêt du transformateur est de pouvoir obtenir un rapport de tension entrée/sortie du convertisseur qui peut devenir grand avec un bon rendement, ce qui n'est pas possible avec une structure non-isolée pour laquelle le rapport cyclique est obligatoirement faible (approximativement dans le rapport des tensions sortie/entrée). Le facteur de forme du courant dans les interrupteurs est défavorable en termes de pertes ; un rapport e/s > 10 est le maximum raisonnable avec une structure nonisolée. Cet aspect conditionne donc certains choix : valeurs des tensions intermédiaires sur les bus DC, localisation de l'isolement, isolements multiples.

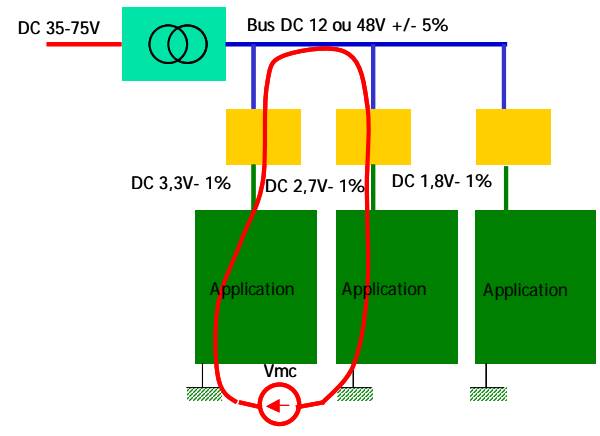

a)

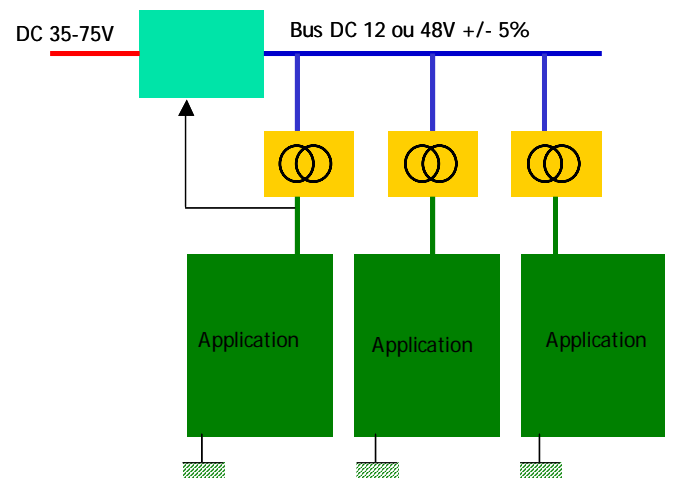

b)

Figure 14 : différentes stratégies de localisation de l’isolement et d'alimentation

Cette organisation permet une grande versatilité de contrôle, celui-ci pouvant être distribué à chaque niveau ou piloté du bas vers le haut (flèche en noir sur la figure 14b)) pour des performances dynamiques particulières. Ce type de système doit fournir une tension continue dont l'ondulation relative doit rester inférieure à $5 \%$ (parfois $2 \%$ ) pour des $d I_{\text {charge }} / d t$ de l'ordre de $1 \mathrm{kA} / \mu \mathrm{s}$ !

\section{4) Structure à commutation douce}

Le concept innovant du module transformateur de tension (Voltage Transformer Module) a été introduit récemment par 
la société Vicor, il est basé sur l'idée d’utiliser un convertisseur à résonance série fonctionnant en permanence à la résonance, les pertes par commutation sont effectivement nulles et l'énergie de commande des interrupteurs est très faible car la commande est également résonnante. Le réglage du transfert de puissance est assuré par un convertisseur amont. Le schéma de la figure 15 indique le principe de la chaîne de conversion (onduleurtransformateur-redresseur_synchrone). L'entrelacement de 2 convertisseurs découpant à 1,75 MHz est rendu possible par la commande des interrupteurs sans pertes (fermeture en mode ZVS, ouverture en mode ZCS).

En octobre 2004, un module 48V/1,5V/90A a été mis sur le marché. L'excellence du rendement (91\% pour le point nominal) a permis une intégration poussée des convertisseurs, soit une densité de puissance de $35 \mathrm{~kW} /$ litre. Un intérêt particulier de cette solution réside dans l'isolation galvanique apportée par le transformateur.

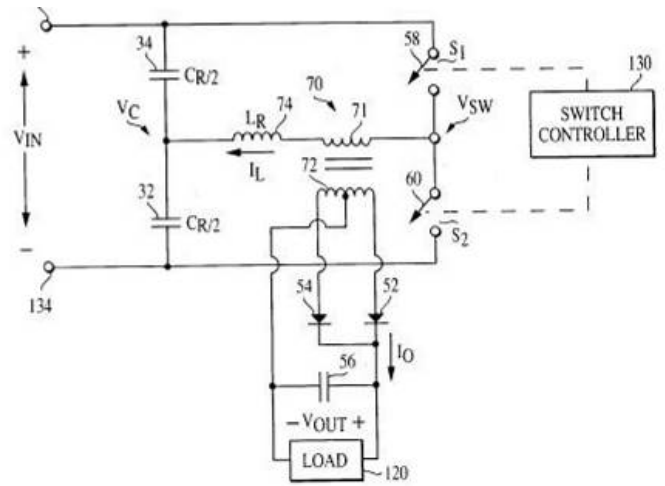

Fig. 15. Structure du module VTM

\section{Commande de grille}

Une structure classique de commande de grille est représentée à la figure 16 . Les transistors T1 et T2 assurent la charge et la décharge de la capacité $C_{g s}$ de grille. La résistance $r$ représente la résistance équivalente du circuit qui est constituée par les diverses résistances du circuit de commande et la grille polycristalline du transistor de puissance. La tension de la source $\mathrm{E}$ doit être égale à la tension $V_{\text {gsmax }}$ à laquelle on souhaite charger la grille.

L'énergie W fournie par la source $E$ vaut donc :

$W=Q_{g s} V_{g s \max }=C_{g s} V_{g s \max }^{2}$

et la puissance :

$P=F_{s w} C_{g s} V_{g s \max }^{2}$

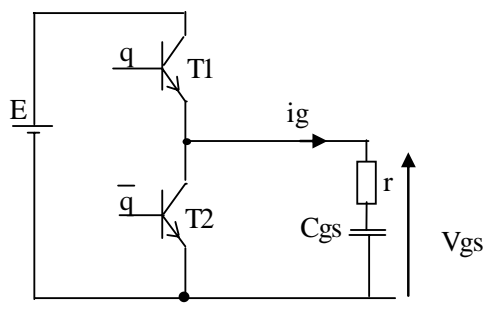

Fig. 16. Structure classique de commande de grille

La commande résonnante est basée sur l'idée d’échanger à chaque commutation l'énergie stockée dans $C_{g s}$ avec une inductance, ce qui permet (si l'amortissement est faible) d'inverser la tension lors du blocage et de diminuer le prélèvement d'énergie sur la source $E$ '.

La structure et les formes d'onde caractéristiques sont données à la figure 17.

Il faut noter que la tension $V^{+}$doit être égale à $V_{g s m a x}$ et que la tension $E$ ' n'est plus nécessairement égale à cette valeur.

On peut montrer que l'expression du courant $i_{s}$ prélevé (cas1) sur la source E' s'exprime par :

$$
i_{s}(t)=-C_{g s} \omega_{o}\left(V^{-}-E^{\prime}\right) e^{m \omega_{o} t} \sin \left(\omega_{o} t\right)\left(1+m^{2}\right)
$$

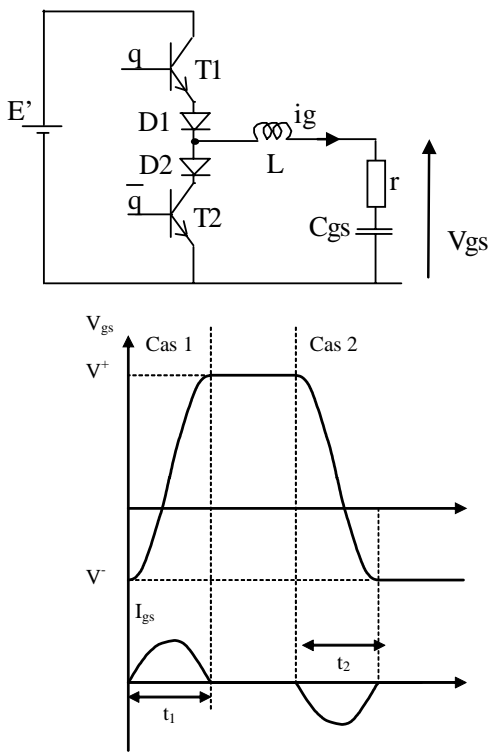

Figure 17 : commande de grille résonnante et forme d'onde caractéristique avec :

$$
\begin{aligned}
& \omega_{o}=\frac{1}{\sqrt{L C_{g s}}} \sqrt{1-m^{2}} m=\frac{r}{2} \sqrt{\frac{C_{g s}}{L}} \\
& \text { et }: V^{-}=-\frac{E}{m \pi} \quad V^{+}=V_{g s \max }=\frac{E}{m \pi}
\end{aligned}
$$

Si l'amortissement $m$ est petit, on en déduit l'énergie $W$ ' prélevée à la source durant la demi-oscillation $\mathrm{t}_{1}$ :

$$
W^{\prime}=2 C_{g s}\left(V_{g s \max } m \pi\right)^{2}\left(\frac{1}{m \pi}+1\right)\left(1+m^{2}\right)
$$

Ainsi, le gain $\kappa$ sur les énergies prélevées dans les deux configurations de commande est-il :

$$
\kappa=\frac{W}{W^{\prime}}=\frac{1}{2 m \pi(m \pi+1)\left(1+m^{2}\right)} \approx \frac{1}{2 m \pi(m \pi+1)}
$$

A titre d'illustration, pour $r=5 \Omega, C_{g s}=2 \mathrm{nF}, L=3 \mu \mathrm{H}$, on obtient : $m=0,065$, pour $V_{\text {gsmax }}=15 \mathrm{~V}$ il faut $E^{\prime}=3 \mathrm{~V}$ et $\kappa=2$. On divise ainsi par 2 la consommation de la commande à partir d'une source à plus basse tension. Les interrupteurs fonctionnent en commutation à zéro de courant et présentent donc des pertes réduites, toutefois, le blocage des diodes D1 et D2 avec des gradients de courant élevés occasionne une dégradation des performances.

Des travaux récents [11] ont permis la mise au point d'une commande à très faibles pertes basée sur l'idée de fournir le courant juste nécessaire à la charge de la grille à $V_{\text {gsmax }}$ dans 
une durée imposée. Le schéma de cette structure et les formes d'onde caractéristiques sont représentées à la figure 18. Le principe consiste à faire croitre le courant dans une inductance puis à transférer l'énergie stockée au secondaire afin d'assurer la charge (décharge) de la capacité de grille. L'intérêt de cette structure réside dans le fonctionnement à zéro de tension des deux interrupteurs, elle présente donc de très faibles pertes.
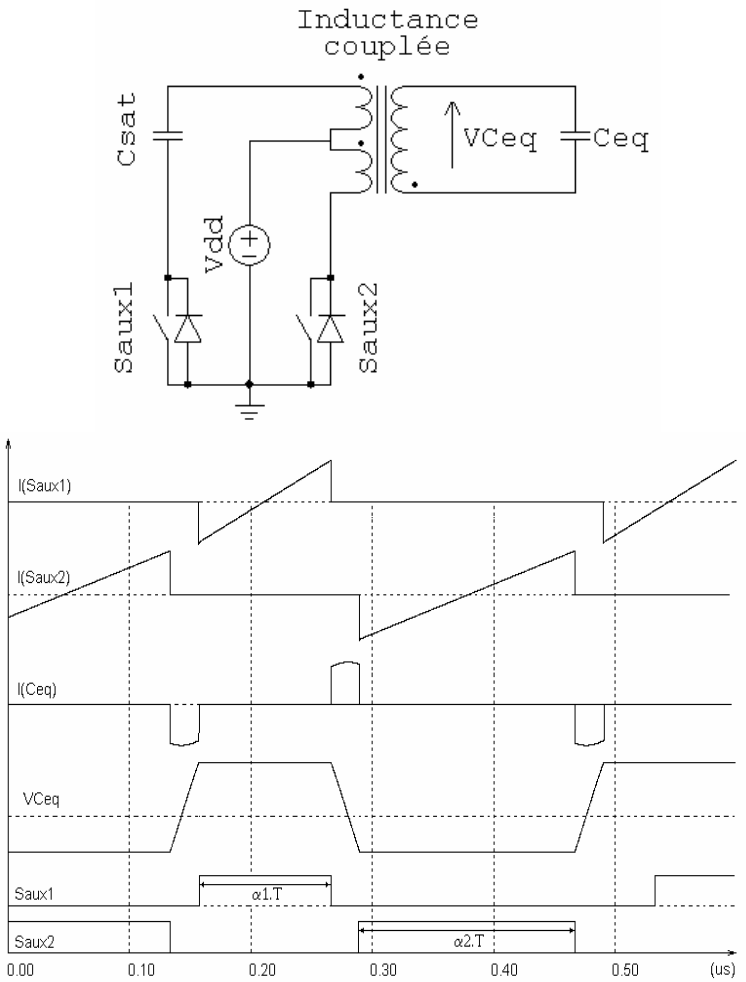

Fig.18. Commande à impulsions de courant

\section{TECHNOLOGIE DES COMPOSANTS}

Le choix technologique des composants et les performances des matériaux qui les composent déterminent directement les pertes, on choisit des composants qui présentent les valeurs les plus faibles possibles de ces paramètres. Leur packaging a aussi un rôle déterminant comme on le verra ci-dessous.

\section{1) Pertes dépendantes de la technologie des transistors à grille isolée}

Dans l'exemple précédent de l'absorbeur sinusoïdal (fig.2), quatre technologies d'interrupteurs différentes (2 IGBT et 2 MOSFET) ont été testées, toujours toutes choses égales par ailleurs [1]. La figure 19 permet de comparer en fonction de la technologie du transistor les pertes dans le couple transistor-diode selon la puissance de sortie.

Les courbes de pertes dans les transistors de la figure 19a) montrent l'avantage net des MOSFET sur les IGBT et notamment du COOLMOS. L'écart de pertes des deux MOSFET est à attribuer en partie aux pertes par conduction (rapport 2 sur $\left.r_{d s o n}\right)$ et aux pertes d'amorçage ( $C_{I S S}$ de $30 \%$ supérieure pour l'IRFP460 de technologie ancienne et présentant plus de cellules en parallèle).

Concernant les deux IGBT, on peut remarquer que le SGW15N60 de technologie NPT récente, est plus dissipatif à bas niveaux de courant que le composant ultra-fast IRG4PC30U de la génération précédente. Concernant la diode rapide (figure 19b), on relève un nuage de points très proches d'une droite moyenne.

Les pertes sont donc fortement dépendantes du courant moyen qui traverse la diode.

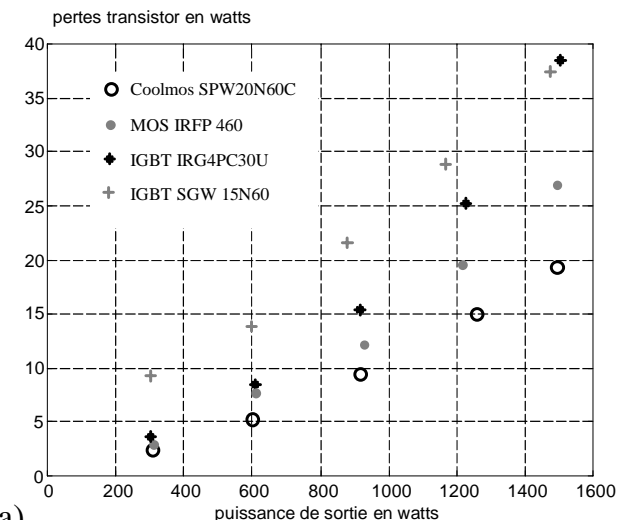

(a)

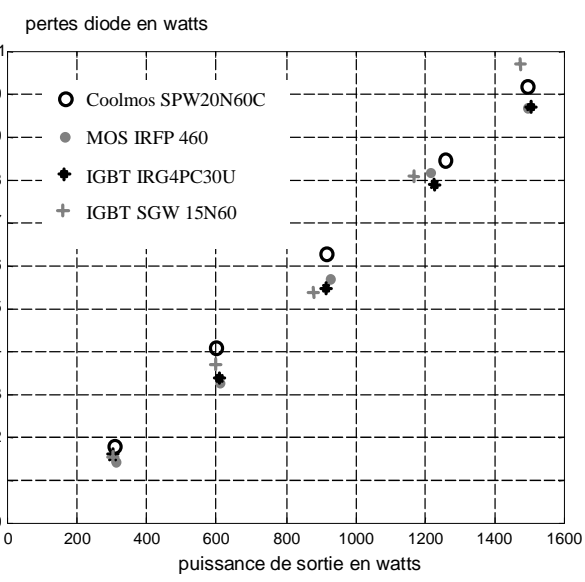

(b)

Figure 19 : pertes dans le transistor (a) et la diode (b) de la structure BOOST selon la technologie

L'écart sur les gradients de courant au blocage selon le transistor utilisé, modifie relativement peu les pertes correspondantes.

Si on appelle $t_{b}$ le temps nécessaire au courant de recouvrement inverse $I_{R R M}$ pour revenir à zéro, les pertes au blocage s'expriment par la relation :

$W_{b}=U_{d c} \frac{I_{R R M} t_{b}}{2}$

Les commutations rapides s'accompagnent d'un accroissement de $I_{R M}$ mais dans le même temps d'une diminution de $t_{b}$. Pour les valeurs de gradients expérimentés avec nos composants, il apparaît un effet quasi compensateur ; la nature technologique des transistors est donc sans effet notable sur les pertes de la diode.

\section{2) Connectique et packaging des transistors MOSFET}

Le packaging des semi-conducteurs devient essentiel dans le contexte de la montée en fréquence de découpage, de la miniaturisation des équipements et de la réduction des pertes. En effet, il devient impératif de maîtriser les inductances parasites qui contribuent aux pertes en commutation, à la 
génération de régimes parasites et à l'émission de perturbations électromagnétiques (CEM).

Par ailleurs, le boîtier des semi-conducteurs doit permettre d'abaisser les résistances de connexion et de faciliter le drainage thermique. La figure 20 présente l'évolution sur le packaging des transistors MOSFET depuis quelques années et l'amélioration des performances obtenues en termes de résistance et d'inductance parasite [12].

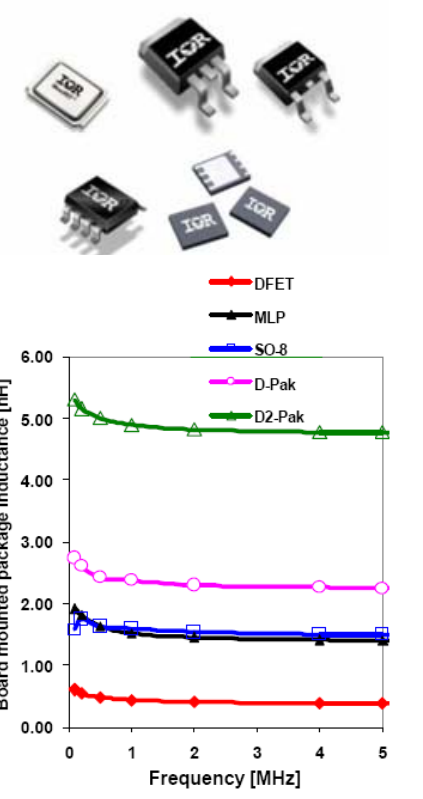

Rangée du haut: DirectFET ${ }^{\mathrm{TM}}$, D2PAK, DPAK

rangée du bas : SO8, MLP

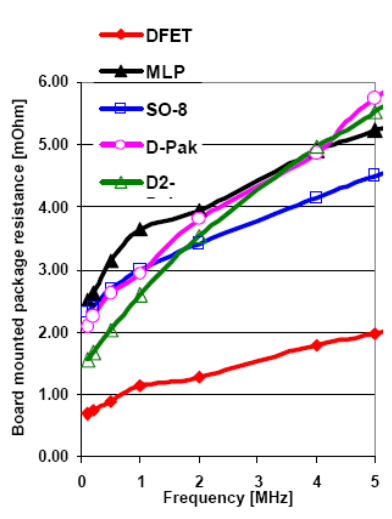

Figure 20 : évolution des performances du packaging (d’après [11])

La connectique imprimée (PCB ou SMI (substrat métallique isolé) doit suivre également la même évolution, la figure 21 présente l'évolution de la résistance et de l'inductance normalisée apparente d'un conducteur imprimé selon son épaisseur et sa constitution en fonction de la fréquence [12].
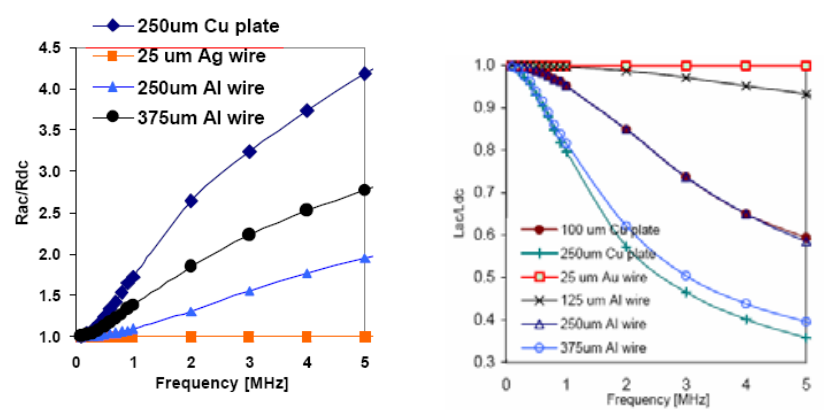

Figure 21 : évolution de la résistance normalisée apparente d'un conducteur imprimé [12]

On constate donc la nécessité d'adapter le choix technologique de la connectique à la gamme de fréquence envisagée.

\section{3) Composants magnétiques}

Une source importante de pertes dans un convertisseur est constituée par les composants magnétiques lorsqu'ils fonctionnent avec une forte dynamique de flux et à fréquence élevée ; c’est le cas des transformateurs dans les alimentations à découpage et/ou des inductances des circuits résonnants. Leurs pertes sont dues aux deux matériaux qui les composent : le cuivre et le matériau magnétique.

a) Pertes par effet Joule

Idéalement, les conducteurs sont dimensionnés de telle sorte que leur densité de courant y soit constante et homogène ; la dissipation de chaleur autorisée par la géométrie du dispositif fixe cette valeur idéale. Des valeurs usuelles comprises entre 3 et $10 \mathrm{~A} / \mathrm{mm}^{2}$ sont généralement admises, ce qui conduit à une valeur minimale de la résistance apparente. Or, dans la fenêtre du composant magnétique, les enroulements subissent un champ électromagnétique à haute fréquence qui provoque une modification spatiale de leur densité de courant (effet de peau et de proximité). Ce flux de fuite dépend de la topologie du circuit magnétique, de la présence d'un entrefer, de la forme des conducteurs, de leur positionnement dans la fenêtre et de la fréquence. Il en résulte que la résistance apparente (et les pertes) peuvent varier considérablement par rapport au cas idéal selon ces considérations. L'exemple suivant [13] illustre ces effets. Les simulations par éléments finis de la figure 22 présentent la répartition du flux magnétique dans un transformateur d'alimentation Flyback fonctionnant à $20 \mathrm{kHz}$ (présence d'un entrefer qui crée des fuites magnétiques) ainsi que la densité de courant dans les enroulements, pour deux dispositions d'enroulement : à proximité (cas 1) et éloigné (cas 2) de l'entrefer. Les enroulements primaires et secondaires sont imbriqués par couche. Lorsque les conducteurs sont près de l'entrefer, on peut noter des valeurs élevées de la densité de courant et de fortes inhomogénéités qui conduisent à des valeurs très différentes de la résistance apparente, elle est multipliée par 2,5 (ainsi que les pertes à même courant) dans cet exemple.
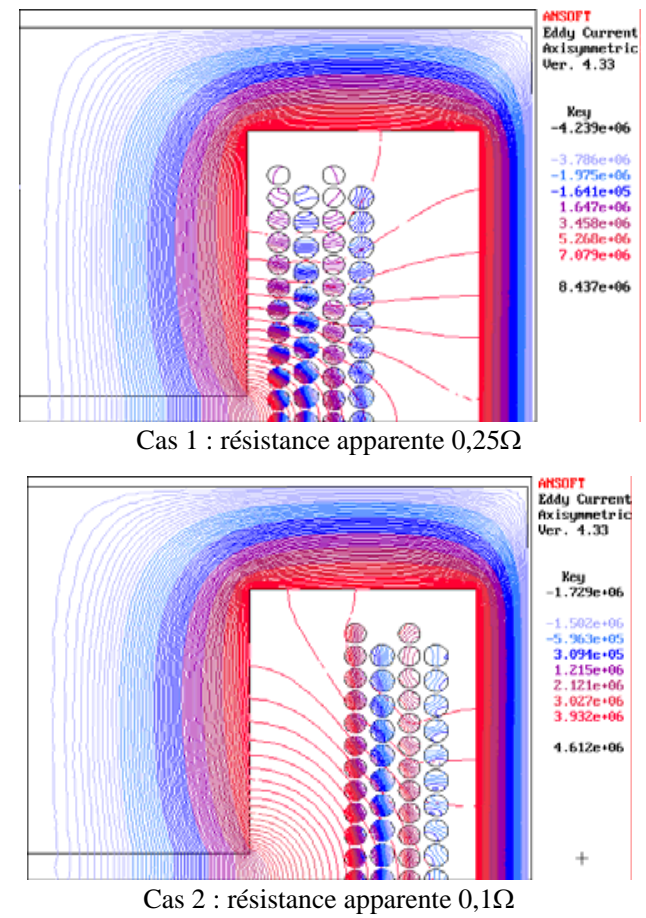

Figure 22 : répartition du flux d'induction et de la densité de courant dans les conducteurs d'un transformateur Flyback (d'après [12]) selon leur positionnement dans la fenêtre, $\mathrm{F}=20 \mathrm{kHz}$ 
La figure 23 présente les courbes d'évolution de la densité de courant $J$ dans les conducteurs horizontaux en vis-à-vis de l'entrefer ainsi que du champ magnétique $H$ selon l'axe passant par le centre de l'entrefer (bord inférieur des figures 22). On constate bien des évolutions très différentes de ces grandeurs selon le positionnement des conducteurs qui conduisent à des pertes accrues d'un facteur 2,5.

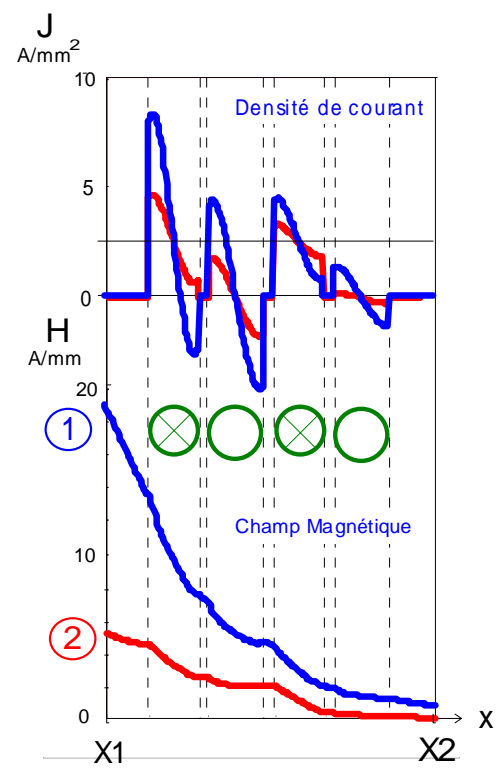

Figure 23 : répartition de la densité de courant et du champ dans les conducteurs en vis-à-vis de l'entrefer selon le positionnement du bobinage dans la fenêtre

Cet exemple illustre la nécessité d'adapter au mieux la topologie du bobinage et la nature des conducteurs (ronds ou plats) selon le régime de champ de fuite du dispositif si on veut minimiser les pertes.

\section{b) Pertes magnétiques}

Elles constituent la seconde source de pertes dans un composant magnétique, elles dépendent essentiellement du matériau, son choix est donc d'importance. Il est intéressant d'utiliser le facteur de mérite du matériau : il est défini par le produit $\Lambda=F \cdot B_{\max }$ qui conduit à une densité de pertes donnée dans le matériau. On sait que le dimensionnement d'un composant magnétique fait appel au produit des aires $A_{p}$ (section de fer $x$ section bobinable), ainsi pour un transformateur on a :

$$
A_{p} \propto \frac{P_{\text {transmise }}}{\Lambda \cdot J}
$$

Pour réduire le volume du composant à densité de pertes fixée on cherchera donc le matériau qui présente la valeur de $\Lambda$ la plus grande à la fréquence considérée. La figure 24 présente quelques courbes d'évolution de cette grandeur en fonction de la fréquence $F$ pour différents matériaux. Toutefois, il apparaît sur cette courbe que le matériau qui présente les valeurs de $\Lambda$ les plus grandes à faible fréquence est le plus intéressant car il permet d'assurer la contrainte de volume tout en minimisant les pertes dépendantes de la fréquence. Ainsi, le matériau nanocristallin (SNC) est-il intéressant de ce point de vue [14].

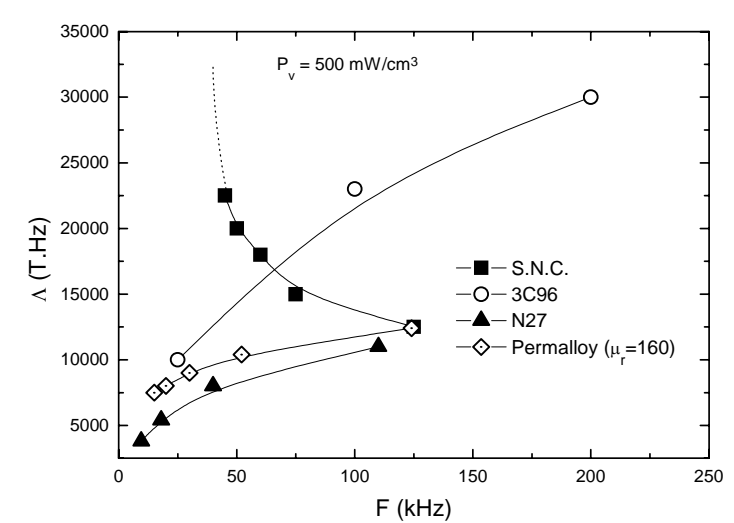

Figure 24 : évolution du facteur de mérite $\Lambda$ pour différents matériaux magnétiques

\section{CONCLusion}

Cet article a montré que la conception d'une alimentation à découpage haute fréquence à haut rendement sur une large plage de charge nécessite d'abord un choix d'architecture de conversion dicté par les conditions d'usage. Une analyse globale des pertes dans la structure selon ses conditions de fonctionnement permet de hiérarchiser l'origine des pertes et d'optimiser les moyens de les minimiser par un choix adéquat des fonctions et de tous les composants (commande, technologie des composants, matériaux).

\section{REFERENCES}

[1] A. Cunière, F. Costa, « Absorbeur sinusoïdal : étude du rendement et des perturbations conduites. », Journal 3EI, 06/2002, N²9, pp.37-47.

[2] Barbi and al. "A $3 \mathrm{~kW}$ unity power factor rectifier based on a two cell boost converter using a new parallel connection technique" ; IEEE transactions on power electronics janvier 1999, volume14, $\mathrm{N}^{\circ} 1$.

[3] Ch. Crebier "Intégration monolithique de composants de puissance" habilitation à diriger des recherches, Université J. Fourier, mai 2006, Grenoble.

[4] http://developer.intel.com/design/pentium4/guides/249205.htm.

[5] Scott Deuty, HDTMOS Power MOSFETs Excel in Synchronous Rectifier Applications, note d'applications AN1520/D, ON semiconductor.

[6] Kaiwei Yao, "High frequency and High performance VRM design for the next generations of Processors”, Virginia Tech PhD thesis, 2005.

[7] F. Forest, T. Meynard, E. Labouré, V. Costan, A. Cunière, T. Martire, "Optimization of the Supply Voltage System in Interleaved Converters Using Intercell Transformers”, IEEE Transactions on Power Electronics, vol. 22, no. 3, pp. 934-942, May 2007.

[8] Yuri Panov and Milan M. Jovanovic, “Design and Performance Evaluation of Low-Voltage/High-Current DC/DC On-Board Modules”, IEEE transactions on Power Electronics, Vol. 16, $\mathrm{N}^{\circ} 1$, janv. 2001, pp.26-33.

[9] F. Wilmot, "contribution à la conception et au dimensionnement de fonctions passives intégrées ", thèse de l’ENS de Cachan, 17 déc. 2004.

[10] http://www.artesyn.com/powergroup/pol_special_vrm.htm.

[11] JM. Jarrousse, E. Labouré, F. Costa, P. Thomas, «Commande à récupération de charge pour une alimentation résonnante $3 \mathrm{MHz}$. » congrès EPF06, Grenoble juillet 2006.

[12] M. Pavier, A. Sawle, A. Woodworth, R. Monteiro, J. Chiu, C. Blake, "High frequency DC:DC power conversion: The influence of package parasitics“,0-7803-7768-0/03/\$17.00 (c) 2003 ieee, pp.699-704.

[13] Serge Delbosc, "Etude de convertisseurs AC/DC à absorption sinusoïdale" ; thèse de doctorat de l'ENS CACHAN, 1999.

[14] F. Costa, F. Alves, A. Benchabi, F. Simon, S. Lefebvre, "Design of a Flyback transformer using a stress-annealed iron-based nanocrystalline alloy”, EPJ AP N¹8, pp.173-180 (2002). 


\section{Biographie des auteurs}

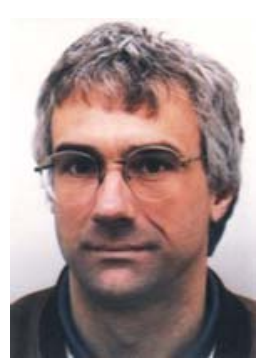

François Costa, est né le 14 décembre 1958, docteur de l'université d’Orsay (1992), habilité à diriger des recherches depuis 1998. Depuis 2003, il est professeur des universités et directeur adjoint chargé des formations technologiques et professionnelles à l'IUFM de Créteil, université Paris 12, où il contribue à la formation des enseignants des Lycées.

Il est actuellement responsable de l'équipe IPEM (Intégration de Puissance et Matériaux) à SATIE. Ses travaux portent sur la CEM des systèmes complexes de conversion d'énergie, sur l'intégration des composants passifs, sur l'utilisation des matériaux piézoélectriques en électronique de puissance et sur la métrologie des courants et l'instrumentation dans ces domaines.

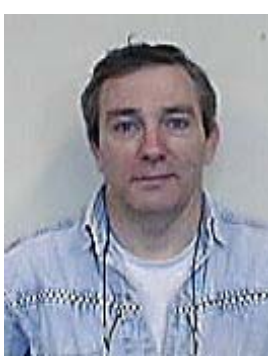

Alain Cunière, né le 24 mars 1956 est professeur agrégé de génie électrique au lycée Coubertin de Meaux (77). Il a obtenu le DEA de génie électrique de l'université Paris XI en 2001 et depuis collabore avec le SATIE sur les problèmes de conversion d'énergie à haut rendement. Il dispense également un cours d'électronique de puissance à l'école supérieure de conception et de production industrielles (ESCPI du CNAM) et participe à la préparation des enseignants des lycées au concours de l'agrégation interne de génie électrique. Ces activités l'ont amené à réfléchir sur les méthodes d'enseignement de l'électronique de puissance. Actuellement, il mène une thèse sur la conversion d'énergie appliquée à la génération de très basses tensions continues. 